\title{
Blockade of the swelling-induced chloride current attenuates the mouse neonatal hypoxic-ischemic brain injury in vivo
}

Raymond WONG ${ }^{1,2}$, Ahmed ABUSSAUD ${ }^{1,2}$, Joseph WH LEUNG ${ }^{1,2}$, Bao-feng XU ${ }^{1,2}$, Fei-ya LI ${ }^{1,2}$, Sammen HUANG ${ }^{1,2}$, Nai-hong $\mathrm{CHEN}^{5}$, Guan-lei WANG ${ }^{6}$, Zhong-ping FENG ${ }^{2}$, Hong-shuo SUN ${ }^{1,2,3,4, *}$

Departments of ${ }^{1}$ Surgery, ${ }^{2}$ Physiology, ${ }^{3}$ Pharmacology, and ${ }^{4}$ Institute of Medical Science, Faculty of Medicine, University of Toronto, Toronto, Ontario, Canada M5S 1A8; ${ }^{5}$ Institute of Materia Medica, Chinese Academy of Medical Sciences and Peking Union Medical College, Beijing 100050, China; ${ }^{6}$ Department of Pharmacology, Zhongshan School of Medicine, Sun Yat-sen University, Guangzhou 510080, China

\begin{abstract}
Activation of swelling-induced $\mathrm{Cl}^{-}$current $\left(I_{\mathrm{Cl}, \text { swell }}\right)$ during neonatal hypoxia-ischemia $(\mathrm{HI})$ may induce brain damage. Hypoxic-ischemic brain injury causes chronic neurological morbidity in neonates as well as acute mortality. In this study, we investigated the role of $I_{\text {Cl,swell }}$ in hypoxic-ischemic brain injury using a selective blocker, 4-(2-butyl-6,7-dichloro-2-cyclopentylindan-1-on-5-yl) oxybutyric acid (DCPIB). In primary cultured cortical neurons perfusion of a $30 \%$ hypotonic solution activated $I_{\mathrm{Cl} \text {,swell }}$, which was completely blocked by the application of DCPIB $(10 \mu \mathrm{mol} / \mathrm{L})$. The role of $I_{\mathrm{Cl}, \text { swell }}$ in neonatal hypoxic-ischemic brain injury in vivo was evaluated in a modified neonatal hypoxic-ischemic brain injury model. Before receiving the ischemic insult, the mouse pups were injected with DCPIB (10 mg/ $\mathrm{kg}$, ip). We found that pretreatment with DCPIB significantly reduced the brain damage assessed using TTC staining, Nissl staining and whole brain imaging, and improved the sensorimotor and vestibular recovery outcomes evaluated in neurobehavioural tests (i.e. geotaxis reflex, and cliff avoidance reflex). These results show that DCPIB has neuroprotective effects on neonatal hypoxic-ischemic brain injury, and that the $I_{\mathrm{Cl}, \text { swell }}$ may serve as a therapeutic target for treatment of hypoxic-ischemic encephalopathy.
\end{abstract}

Keywords: neonatal stroke; neonatal hypoxic-ischemic brain injury; volume regulated anion channels; chloride channels; cortical neurons; whole-cell recording; DCPIB; neurobehavioural tests; neuroprotection

Acta Pharmacologica Sinica (2018) 39: 858-865; doi: 10.1038/aps.2018.1; published online 29 Mar 2018

\section{Introduction}

Neonatal hypoxic-ischemic (HI) brain injury is a condition that occurs when the brain is deprived of adequate oxygen ${ }^{[1,2]}$. In infants and children, HI brain damage is a major cause of acute mortality and chronic neurological disability, such as hypoxic-ischemic encephalopathy $(\mathrm{HIE})^{[3]}$. The leading cause of $\mathrm{HI}$ brain injury is birth asphyxia, which causes $23 \%$ of all neonatal deaths worldwide ${ }^{[4,5]}$. Although the prevalence of asphyxia in full-term newborn infants is $\sim 0.2 \%$ (2-4 per 1000), it reaches $\sim 60 \%$ in premature or low birth weight newborns. Mortality rate is high at $\sim 20 \%$ to $50 \%$ of those afflicted. Furthermore, survivors suffer from severe motor impairments and learning disabilities ${ }^{[2,5]}$. This is in part because HI brain

\footnotetext{
*To whom correspondence should be addressed.

E-mail hss.sun@utoronto.ca

Received 2017-11-07 Accepted 2018-01-02
}

injury often results in HIE and/or cerebral palsy, a generic term describing a group of perceptive, cognitive, and motor disorders resulting from damage to or abnormal development of the brain ${ }^{[6]}$. Children with cerebral palsy have affected neurological functions (eg vision, auditory, etc), and depending on the severity, early detection of cerebral palsy can be challenging $^{[6-8]}$. Currently, there is no cure available; standard therapeutic strategies are aimed at assisting patients with cerebral palsy to develop into their full potential of independency ${ }^{[7,8]}$. However, lifelong rehabilitation is often necessary ${ }^{[7,8]}$. Therefore, survivors of infant HI brain injury suffer lasting neurological damage, which negatively influences their quality of life. Moreover, the lifetime cost to the healthcare system is $\sim \$ 1.5$ million per person in Canada ${ }^{[9]}$. Thus, HI brain injury and HIE represent a serious socioeconomic burden, and there is urgent need to elucidate the underlying mechanisms in order to develop a more effective prevention and treatment. 
However, the mechanisms underlying neonatal HI remain illdefined.

An important cellular role of the volume-regulated anion channels (VRACs), a major class of ubiquitously expressed $\mathrm{Cl}^{-}$channels, is the regulation of cell volume ${ }^{[10]}$. This is accomplished through the efflux of $\mathrm{Cl}^{-}$when the cell experiences hypo-osmotic swelling ${ }^{[10,11]}$. Consequently, VRACs mediate the swelling-induced $\mathrm{Cl}^{-}$current $I_{\mathrm{Cl} \text {,swell }}$. This has major implications on cell volume homeostasis (ie to decrease cell volume during osmotic perturbations). VRACs are highly expressed in the brain, and the swelling-induced chloride current $I_{\mathrm{Cl} \text {,swell }}$ has been suggested to be involved in cerebral ischemia ${ }^{[10,12]}$. Under physiological conditions, hypo-osmotic swelling activates swelling-induced chloride current $I_{\mathrm{Cl} \text {,swell }}$ via mechanosensory mechanisms (that is still poorly understood), which results in $\mathrm{Cl}^{-}$efflux. This efflux is coupled with $\mathrm{K}^{+}$efflux, which leads to the expulsion of water osmotically ${ }^{[13]}$. As a result, cell volume is reduced, thus retaining homeostasis. Nonetheless, it should be noted that the molecular identities of the channels underlying the swelling-induced $\mathrm{Cl}^{-}$current $I_{\mathrm{Cl} \text {,swell }}$ remain controversial $^{[13]}$. Despite this, however, there is evidence demonstrating that 4-(2-butyl-6,7-dichloro-2-cyclopentyl-indan-1-on-5-yl) oxobutyric acid (DCPIB) is a selective antagonist that potently inhibits the swelling-induced $\mathrm{Cl}^{-}$current $I_{\mathrm{Cl} \text {,swell }}{ }^{[14]}$. Under pathological conditions, prolonged neuron overstimuation (eg by glutamate) results in excitotoxicity that causes excessive depolarization ${ }^{[15]}$. This, in turn, drives water inflow and consequently results in cell swelling ${ }^{[15]}$. Due to the persistent depolarization, there is influx of $\mathrm{Cl}^{-}$(rather than efflux) through the swelling-induced chloride current $I_{\mathrm{Cl} \text {,swell }}$ (ie, opposite phenomenon compared to the normal regulatory volume decrease mechanism under physiological conditions). Consequently, a positive feedback for further swelling is established, and ultimately necrotic neuronal death is induced.

Because swelling-induced $\mathrm{Cl}^{-}$current $I_{\mathrm{Cl} \text {,swell }}$ is normally inactive ${ }^{[15]}$, the rationale is that pharmacologically reducing swelling-induced $\mathrm{Cl}^{-}$current $I_{\mathrm{Cl} \text {,swell }}$ activity would not affect normal brain function. Thus, swelling-induced $\mathrm{Cl}^{-}$current $I_{\mathrm{Cl} \text {,swell }}$ is a suitable potential drug target for treatment of HI brain injury, and inhibition of swelling-induced $\mathrm{Cl}^{-}$current $I_{\mathrm{Cl} \text {,swell }}$ is a feasible therapeutic strategy. Previous publication described the role of the swelling-induced $\mathrm{Cl}^{-}$current $I_{\mathrm{Cl} \text {,swell }}$ in mediating adult stroke damage ${ }^{[16]}$. The authors found that pharmacologically inhibiting the swelling-induced $\mathrm{Cl}^{-}$current $I_{\mathrm{Cl} \text {,swell }}$ with DCPIB endowed neuroprotection by reducing infarction and improving neurobehavioural scores in a rat transient middle cerebral artery occlusion (MCAO) model when the drug was delivered intracisternally ${ }^{[16]}$. In our laboratory, we previously described the pharmacological role of DCPIB in reducing HI brain injury in $v i v{ }^{[17]}$, and suppressing glioblastoma cellular functions in vitro by inhibiting the swelling-induced $\mathrm{Cl}^{-}$current $I_{\mathrm{Cl} \text {,swell }}{ }^{[18]}$. Additionally, we also reported that pharmacologically inhibiting the swelling-induced $\mathrm{Cl}^{-}$current $I_{\mathrm{Cl} \text {,swell }}$ in vitro with DCPIB decreased the hypotonic-inducing intracellular $\mathrm{Cl}^{-}$ concentration, and reduced the oxygen and glucose deprivation (OGD)-induced cell death in PC12 cell line ${ }^{[17]}$. Moreover, we showed preliminary evidence that the swelling-induced $\mathrm{Cl}^{-}$

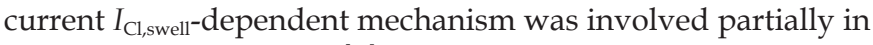
neonatal $\mathrm{HI}$ brain injury ${ }^{[17]}$. However, there is currently no in vivo behavioural assessment demonstrating whether inhibition of the swelling-induced $\mathrm{Cl}^{-}$current $I_{\mathrm{Cl} \text {,swell }}$ by DCPIB can confer neuroprotection at the level of neurobehavioural performance. Here, we show that: 1) in vitro, DCPIB inhibits the swellinginduced $\mathrm{Cl}^{-}$current $I_{\mathrm{Cl} \text {,swell }}$ in cortical neurons; and 2) in vivo, inhibiting the swelling-induced $\mathrm{Cl}^{-}$current $I_{\mathrm{Cl} \text {,swell }}$ with DCPIB treatment results in reduced brain damage, and improved short-term neurobehavioural performance following the $\mathrm{HI}$ insult. Thus, the swelling-induced $\mathrm{Cl}^{-}$current $I_{\mathrm{Cl} \text {,swell }}$-mediated outcomes represent a non-glutamate stroke mechanism that plays critical role in both adult and neonatal variations of multiple brain diseases. There is broad implication because, ultimately, the goal of our work is contribution towards the development of effective therapeutic neuroprotective agents for reducing HI brain injury and its related HIE.

\section{Materials and methods Reagents}

All reagents, unless specified otherwise, were from SigmaAldrich (Canada). DCPIB (Sigma catalogue \# D4068) was first dissolved in $0.2 \%$ dimethyl sulfoxide (DMSO), and then diluted in $1 \%$ phosphate buffered saline (PBS) for the final concentration of $10 \mathrm{mg} / \mathrm{kg}$.

\section{Animals}

CD1 timed-pregnant mice were purchased from Charles River Laboratories (Sherbrooke, Quebec, Canada). Each cage only housed one nursing female mouse and her litter. Food and water were readily accessible, and maintained in a room with an ambient temperature of $20 \pm 1^{\circ} \mathrm{C}$ and a $12: 12 \mathrm{~h}$ light/dark cycle. Experiments involving animals followed the guidelines of the Canadian Council on Animal Care (CCAC) protocol. All animal experimental protocols were approved by the local Animal Care and Use Program Committee, Office of the Research Ethics at the University of Toronto.

\section{Surgical preparation}

Prior to surgery, mouse pups were anesthetised with isoflurane. The skin was cleaned with iodine followed by $75 \%$ alcohol before incision. A midline ventral incision was conducted at the anterior neck. DCPIB was given via intraperitoneal administration. A temperature control and heating blanket system (Harvard Apparatus) was used to monitor/maintain body temperature.

\section{Hypoxic-ischemic brain injury model}

Seven-day-old (P7) postnatal CD1 wild-type mouse pups of either sex were used. As previously described ${ }^{[17,19]}$, a modified Rice-Vannucci neonatal adaptation of the Levine procedure was used to induce cerebral HI injury in the mouse pups. Appropriate protocols were undertaken to minimize discomfort and pain of animals throughout the common carotid artery occlusion (CCAO) procedure. In brief, P7 mice (weigh- 
ing $\sim 5$ to $5.5 \mathrm{~g}$ ) were anesthetised with $3 \%$ isoflurane for $3 \mathrm{~min}$ as induction, and then followed by $2 \%$ isoflurane for maintenance during the procedure. We used a stereo dissecting microscope containing ring lens illumination and fiber-optic bifurcation (SMZ-2B Nikon, Japan). After conducting the midline cervical incision $(0.5 \mathrm{~cm})$ and separating the muscles at the frontolateral neck, the right common carotid arteries become exposed. These were separated from the associated sympathetic and vagus nerves, and then occluded via bipolar electrical coagulation (Vetroson V-10 Bi-polar electrosurgical unit, Summit Hill Laboratories, Tinton Falls, NJ, USA). Animal body temperature was maintained with a homeothermic heating blanket. For each pup, the procedure took $\sim 5$ min for each pup. After surgery, wounds were closed and the pups recovered in a $37^{\circ} \mathrm{C}$ incubator for $10 \mathrm{~min}$ (or until regaining consciousness). Afterwards, pups were returned to their dam (at least $90 \mathrm{~min}$ ) for further recovery.

To induce hypoxia, pups were placed into an airtight chamber (A-Chamber A-15274 with ProOx 110 Oxygen Controller/ E-720 Sensor, Biospherix, NY, USA), into which a humidified gas mixture (7.8\% oxygen balanced with $92.2 \%$ nitrogen) was perfused. Flow of gas was constant for $60 \mathrm{~min}$. A compact oxygen controller (ProOx 110 controller, Biospherix, NY, USA) with a compressed nitrogen gas source (Linde, Mississauga, $\mathrm{ON}$, Canada) was used to regulate oxygen concentration. A homeothermic blanket control unit (K-017484 Harvard Apparatus, Massachusetts, USA) monitored and maintained animal body temperature at $36.5^{\circ} \mathrm{C}$. After exposure to hypoxia, pups recovered on a heating pad and then returned to their dam.

\section{Drug administration}

Mouse pups weighing $\sim 5 \mathrm{~g}$ were randomly assigned into sham group, control HI group or DCPIB-treated HI group. DCPIB $(10 \mathrm{mg} / \mathrm{kg}$ ) with $0.2 \%$ DMSO (DCPIB-treated HI group) or $0.2 \%$ DMSO alone (control HI group) was diluted in $1 \%$ PBS before intraperitoneal injection into the mouse pups. Drug injection was done $\sim 20 \mathrm{~min}$ before CCAO for all tests listed below except for the TTC test ( 1 $\mathrm{h}$ after CCAO). The total volume injected per mouse pup was $0.1 \mathrm{~mL}$.

\section{Neurobehavioral tests}

Two neurobehavioral tests (ie geotaxis, cliff avoidance reaction) were used to assess the recovery outcomes of the mouse pups on Day 1, Day 3, and Day 7 after the HI insult. These tests are well-documented ${ }^{[20]}$, and were carried out as previously described ${ }^{[21-27]}$. In brief, geotaxis reflex is an automatic, stimulus-bound orientation movement. It is considered to be diagnostic of vestibular and/or proprioceptive function. Here, pups were placed head facing down in the middle of a plane inclined at an angle of $45^{\circ}$, and the latency time for the pup to rotate $180^{\circ}$ (ie, head facing up) was recorded. Cliff avoidance reaction evaluates the integration of exteroceptive input and locomotor output. For this test, pups were placed on the edge of a platform, and the latency time for the pup to remove both paws from the edge by turning away from the cliff was recorded.

\section{Measurement of infarct volume}

TTC staining: $24 \mathrm{~h}$ after the HI episode, mouse pups were sacrificed and the brains were extracted and coronally cut into $\sim 1 \mathrm{~mm}$ sections, which were immersed in 2\% 2,3,5-triphenyltetrazolium chloride (TTC) in $1 \%$ PBS at $37^{\circ} \mathrm{C}$ in the dark (20-30 min). TTC is a redox indicator that can indicate cellular respiration for differentiating between metabolically active and inactive tissues. After stained with TTC, brain slices were scanned and infarct areas were measured, as previously described ${ }^{[24-27]}$. An image analysis system (NIH ImageJ) was used to quantify the ipsilateral and contralateral hemisphere area for each section. Volumes were determined by summing the representative areas in all sections and then multiplying by slice thickness. Edema correction was conducted. Infarction volume was calculated with the following formula: CIV $(\%)=[\mathrm{CHV}-(\mathrm{IHV}-\mathrm{IV})] / \mathrm{CHV}^{*} 100$; where $\mathrm{CIV}$ is corrected infarct volume, $\mathrm{CHV}$ is contralateral hemisphere volume, IHV is ipsilateral hemisphere volume, and IV is infarct volume.

Nissl staining/whole brain imaging: Seven days after the HI episode, whole brain tissue was collected and imaged to reveal morphological changes between the groups, as previously described ${ }^{[24-27]}$. At this point, the infarct areas have undergone liquefactive necrosis. The severity of the brain damage was quantified. The brains were sliced into $\sim 100 \mu \mathrm{m}$ coronal sections and stained with 1\% Cresyl violet (Nissl) to indicate histological damage. Infarct area was imaged using ImageJ, and was calculated as follows: IV $(\%)=\mathrm{IV} / \mathrm{CHV}^{\star} 100$.

\section{Primary mouse neuron culture}

Primary cortical neurons were cultured from E16 CD1 mice, as previously described ${ }^{[28]}$. In brief, cortices were isolated and then digested using $0.025 \%$ trypsin/EDTA $\left(37^{\circ} \mathrm{C} ; 15 \mathrm{~min}\right)$. An Improved Neubauer hemocytometer was used to measure cell density. Cells were plated at $1.0 \times 10^{4}$ on poly-D-lysine coated glass coverslips (Sigma; 12 mm № 1 German Glass, Bellco cat. № 1943-10012). Neurons were maintained in serum-free culture Neurobasal medium supplemented with 1.8\% B-27, 0.25\% Glutamax, and $1 \%$ antibiotic-antimyocotic. Incubation of cells was in $5 \% \mathrm{CO}_{2}$ at $37^{\circ} \mathrm{C}$.

\section{Patch clamp electrophysiology}

Recordings were carried out in the whole-cell configuration using the Axopatch 700B (Axon Instruments, Inc), similar to our previous studies ${ }^{[18,29-31]}$. The ramp protocol was from -100 to $+100 \mathrm{mV}(400 \mathrm{~ms} ;-70 \mathrm{mV}$ holding; $5 \mathrm{~s}$ interval at $2 \mathrm{kHz}$; digitized at $5 \mathrm{kHz}$ ). Data acquisition and analyses were via Clampex 9.2 and Clampfit 9.2. All experiments were performed at room temperature. The pipette (intracellular) solution contained (in mmol/L): 145 CsMSF, $8 \mathrm{NaCl}, 55 \mathrm{KCl}, 10$ HEPES, 1 MgATP, and 10 EGTA (pH adjusted to 7.2 with $\mathrm{CsOH}$ ). After filling with solution, pipette resistance was 8-13 M 2 . The bath (extracellular) solution contained (in mmol/L): $140 \mathrm{NaCl}$, $5 \mathrm{KCl}, 2 \mathrm{CaCl}_{2}, 20$ HEPES, and 10 glucose ( $\mathrm{pH}$ adjusted to 7.4 with $\mathrm{NaOH}$; osmolarity to $\sim 300$ mOsm with sucrose). 


\section{Statistic analysis}

GraphPad Prism 6 (GraphPad Software, Inc) was used for statistical analysis. The statistical significance between control and DCPIB-treatment group was assessed with the Student's t-test. In multiple groups, one-way ANOVA with Bonferroni multiple comparison test was used. Significance was defined as $P<0.05$. Data are shown as mean \pm SEM.

\section{Results and discussion}

Since the molecular identities of the swelling-induced $\mathrm{Cl}^{-}$ channels remain elusive, we first measured the electrophysiological properties of the swelling-induced $\mathrm{Cl}^{-}$current $I_{\mathrm{Cl} \text {,swell }}$ in primary neurons and pharmacologically verified with DCPIB (Figure 1). When exposed to $30 \%$ hypotonic solution, there was activation of swelling-induced $\mathrm{Cl}^{-}$current $I_{\mathrm{Cl} \text {,swell }}$, with a reversal potential that is consistent with what we previously reported $^{[18]}$. In the continued presence of the hypotonic solution, application of DCPIB $(10 \mu \mathrm{mol} / \mathrm{L})$ inhibited the activated current (Figure 1A and 1B). As shown in the summary graph (Figure 1C), perfusion of the hypotonic solution activated a swelling-induced $\mathrm{Cl}^{-}$current $I_{\mathrm{Cl} \text {,swell, }}$, which was fully inhibited by subsequent application of DCPIB (ie, hypotonic solution increased current density to $4.05 \pm 0.49 \mathrm{pA} / \mathrm{pF}$ from $1.76 \pm 0.17$ $\mathrm{pA} / \mathrm{pF}, P<0.01$; DCPIB administration abolished this increase by reducing current density back to $1.87 \pm 0.11 \mathrm{pA} / \mathrm{pF}, P<0.01)$. Therefore, the swelling-induced $\mathrm{Cl}^{-}$current $I_{\mathrm{Cl} \text {,swell }}$ is present in primary neurons and sensitive to inhibition by DCPIB.

Next, we provide the first in vivo evidence demonstrating the neuroprotective effects of a selective swelling-induced $\mathrm{Cl}^{-}$ current $I_{\mathrm{Cl} \text {,swell }}$ antagonist DCPIB on improving behavioural functional outcomes after HI brain injury. As shown in the schematic in Figure 2A, we used a well-documented mouse hypoxic-ischemic (HI) model (Rice-Vannucci method) with modifications ${ }^{[17,19]}$. The key events throughout the procedure have been noted on the timeline in Figure 2B. Our model of neonatal $\mathrm{HI}$ brain injury formed a brain infarct that is confined from the ipsilateral brain hemisphere to the common carotid occlusion, as shown from the TTC stain in Figure 3A (white section of the brain; extent of damage $24 \mathrm{~h}$ following $\mathrm{HI}$ ). On the contrary, the contralateral hemisphere did not demonstrate brain injury (red section). This finding is consistent with our previous report ${ }^{[17]}$, noting the reliability of our $\mathrm{HI}$ model. The right hemispheric corrected infarct volume was $46.38 \% \pm 3.76 \%$ $(n=23)$ in animals treated with vehicle (control HI group; Figure $3 \mathrm{~B}$ ). When DCPIB was injected $\sim 1 \mathrm{~h}$ after common carotid artery occlusion (CCAO), the corrected infarct volume was significantly reduced to $31.75 \% \pm 5.14 \%$ (DCPIB-treated HI group; $P<0.05)$. This suggested that inhibition of the swellinginduced $\mathrm{Cl}^{-}$current $I_{\mathrm{Cl} \text {,swell }}$ with DCPIB can reduce the severity of brain damage.

Seven days after the HI, the brains of mouse pups were extracted to examine the extent of morphological and histological damage. As shown in Figure 4, integrity of brain morphology in the control HI group is severely compromised seven days after HI brain injury. However, for animals in the DCPIB-treated HI group, the morphology of these brains is qualitatively more comparable to the brains in the sham group. Consistent with this observation, Nissl staining to assess histological damage also shows a similar trend. That is, the DCPIB-treated HI group showed significantly reduced infarction volume from $41.79 \% \pm 3.23 \%$ to $10.21 \% \pm 2.08 \%$ $(P<0.05 ; n=4$ /group; Figure 4$)$. This is the first evidence to suggest the lasting neuroprotective effect of DCPIB against HI brain injury, as even only one administration of DCPIB 20 min before CCAO elicited an improvement one week after the HI.

We further quantitatively assessed recovery outcomes of the HI by employing short term neurobehavioural tests (ie geotaxis reflex, and cliff aversion reflex). These reflex tests were chosen because they are representative of the earliest development stages in mice, and thus are good indicators of sensorimotor function ${ }^{[20]}$. Specifically, the geotaxis reflex assesses vestibular and proprioceptive functions, whereas the cliff aversion reflex measures the maladaptive impulse behav-
A

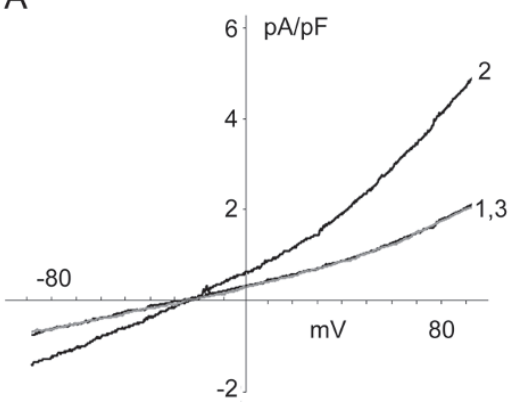

B

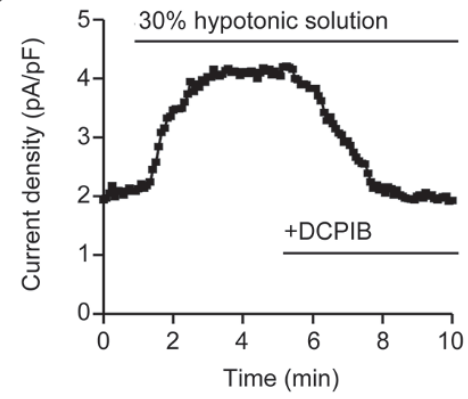

C

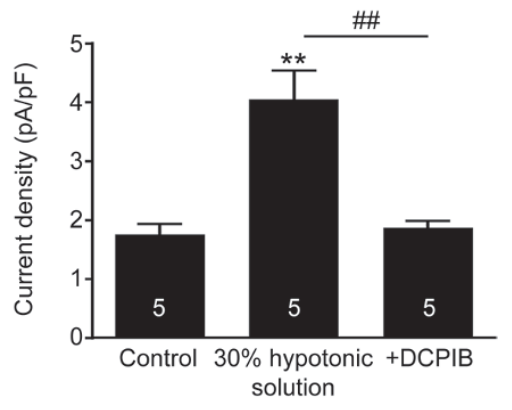

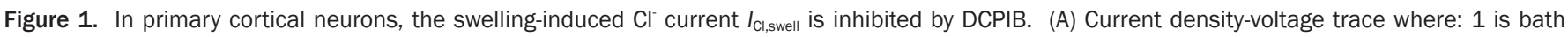

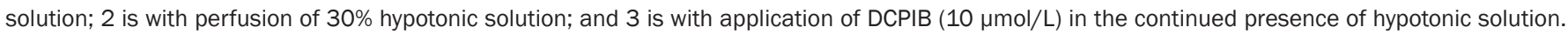

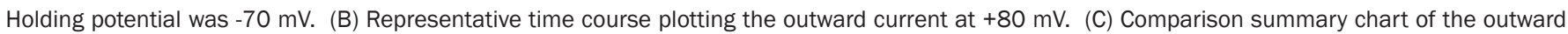

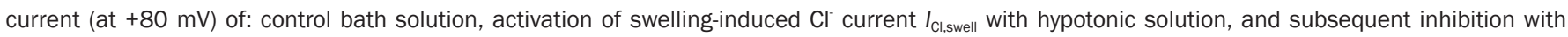

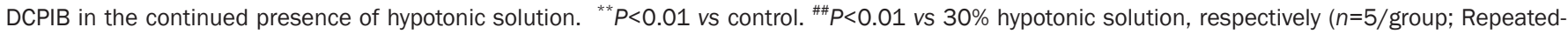
measure One-way ANOVA with Bonferonni multiple comparison test). 
A Neonatal HI mouse model overview

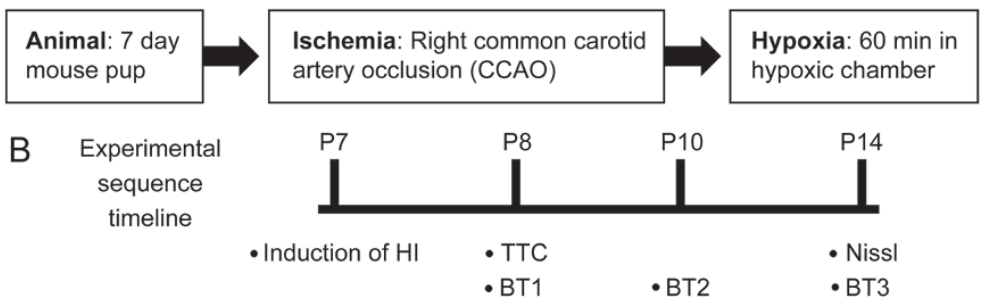

Figure 2. Schematic of the in vivo neonatal hypoxic-ischemic $(\mathrm{HI})$ mouse model to examine the effects of DCPIB. (A) Overview of the process involved in developing the model, which contained two main parts: ischemia and hypoxia. First, using postnatal day 7 (P7) mice, ischemia was induced by isolation of the right common carotid artery and then subsequent ligation with a bipolar electrocoagulation device (common carotid artery occlusion; $\mathrm{CCAO}$ ). Following recovery from the surgery, hypoxia was then induced by placing operated pups in a $37^{\circ} \mathrm{C}$ chamber perfused with a gas mixture of $7.5 \%$ oxygen and $92.5 \%$ nitrogen for $60 \mathrm{~min}$. (B) Timelines of the experimental sequences for model development as well as the subsequent histological, morphological, and behavioural assessments. The timeline was carried out across three groups: sham, control HI, and DCPIB-treated HI. The "control HI group" represents HI-induced animals administered with vehicle only; "DCPIB-treated HI group" represents HI-induced animals treated with DCPIB. Infarct volume measurement with TTC was carried out $24 \mathrm{~h}$ after $\mathrm{HI}$ (ie, when pups were P8), whereas measurement with Niss was 7 days after (ie when pups were P14). Neurobehavioural performance (BT; behavioural test) was assessed at three separate time points; at one, three, and seven days after $\mathrm{HI}$ (ie, when pups were P8, P10, and P14, respectively).
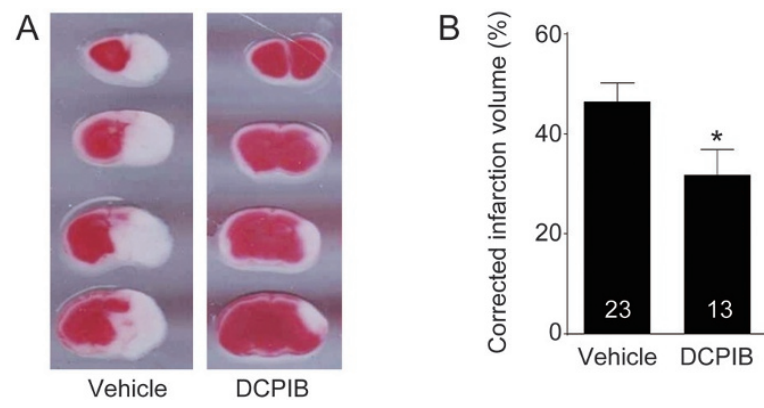

Figure 3. Treatment with DCPIB reduced brain infarct volume of neonatal hypoxic-ischemic (HI) brain injury in vivo. (A) Representative 2,3,5-triphenyltetrazolium chloride (TTC) images of the infarcts of the coronal sections of P8 mouse pup brains treated with vehicle (control $\mathrm{HI}$ group) or $10 \mathrm{mg} / \mathrm{kg}$ DCPIB (DCPIB-treated HI group). (B) Column chart summarizing that animals in the DCPIB-treated HI group $(n=13)$ had significantly reduced brain infarct volume compared to those in the control HI group ( $n=23$; ${ }^{*} P<0.05$; Student's $t$-test).

iour $^{[20]}$. These neurobehavioral tests determine the functional recovery of animals after the insult. A longer time indicates slower reflex, which is considered a negative score (ie, lower time is better). Each test was conducted one, three, and seven days after the HI. Animals in all groups (ie, sham, control HI, and DCPIB-treated HI) were subjected to these tests (Figure 5). On Day 1, pups in the control HI group had the slowest reflex for both geotaxis and cliff avoidance (at $3.61 \pm 0.17$ and $7.42 \pm 0.18 \mathrm{~s}$, respectively; $P<0.05$ for both). Treatment with DCPIB improved reflex speed of animals in the DCPIB-treated HI group (to $2.86 \pm 0.06$ and $5.94 \pm 0.51 \mathrm{~s}$, respectively), which is not significant compared to animals in the sham group $(1.93 \pm 0.27$ and $4.76 \pm 0.31 \mathrm{~s}$, respectively). On Day 3, control HI group animals still had the slowest cliff avoidance reflex, at $6.30 \pm 0.93 \mathrm{~s}(P<0.05)$, which was improved with DCPIB treatment $(3.80 \pm 0.38 \mathrm{~s}$; note that this score was not significantly different compared with the animals in the sham group at
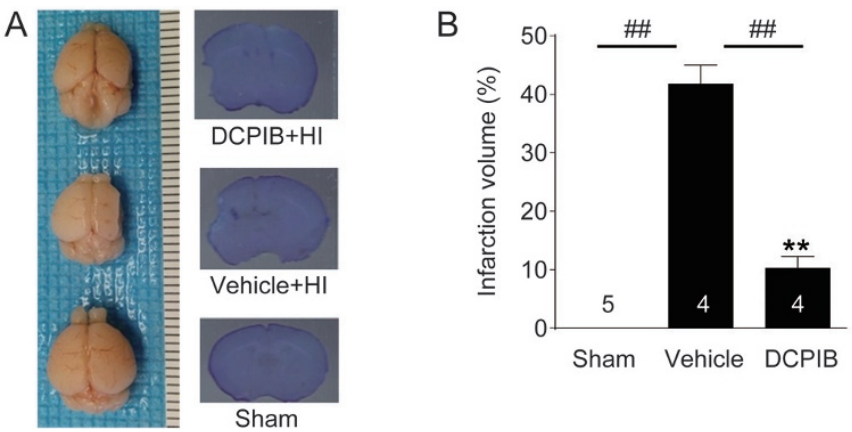

Figure 4. DCPIB-treated animals showed less morphological and histological damage following HI. (A) Left panel shows representative whole brain images of P14 mouse pups in either the sham group (bottom), control HI group (middle), or DCPIB-treated HI group (top). Note that morphological integrity is severely compromised for the control group brain, when compared to the treatment and sham group brains. Right panel displays the corresponding representative images of Nissl staining. (B) Summary column chart showing that intraperitoneal (ip) treatment of DCPIB (10 mg/kg in 0.2\% DMSO) $20 \mathrm{~min}$ before induction of $\mathrm{HI}$ significantly reduced infarct volume measured seven days following the HI episode $\left({ }^{* *} P<0.01\right.$ vs Sham. ${ }^{\# \#} P<0.01$ vs vehicle, respectively; $n=4$ / group; One-way ANOVA with Bonferonni multiple comparison test), thus suggesting the lasting neuroprotective effectiveness of DCPIB.

$2.99 \pm 0.58 \mathrm{~s}$ ). Although pups in the control HI group did not significantly have a slower geotaxis reflex on Day 3, it was clearly gravitating towards this trend. One possible explanation is that at Day 3, mouse pups become P10, which is when their eyes begin to open. The sudden acquisition of vision can potentially confer drastic improvement to vestibular and proprioceptive performance despite suffering HI. This remains to be tested. Nevertheless, by Day 7, geotaxis reflex of the control HI group had markedly worsened. This was ameliorated with inhibition of the swelling-induced chloride current $I_{\mathrm{Cl}, \text { swell }}$ by DCPIB (from $3.15 \pm 0.54 \mathrm{~s}$ in the control HI group to $1.44 \pm 0.14 \mathrm{~s}$ in the DCPIB-treated HI group; $P<0.05$ ). However, 

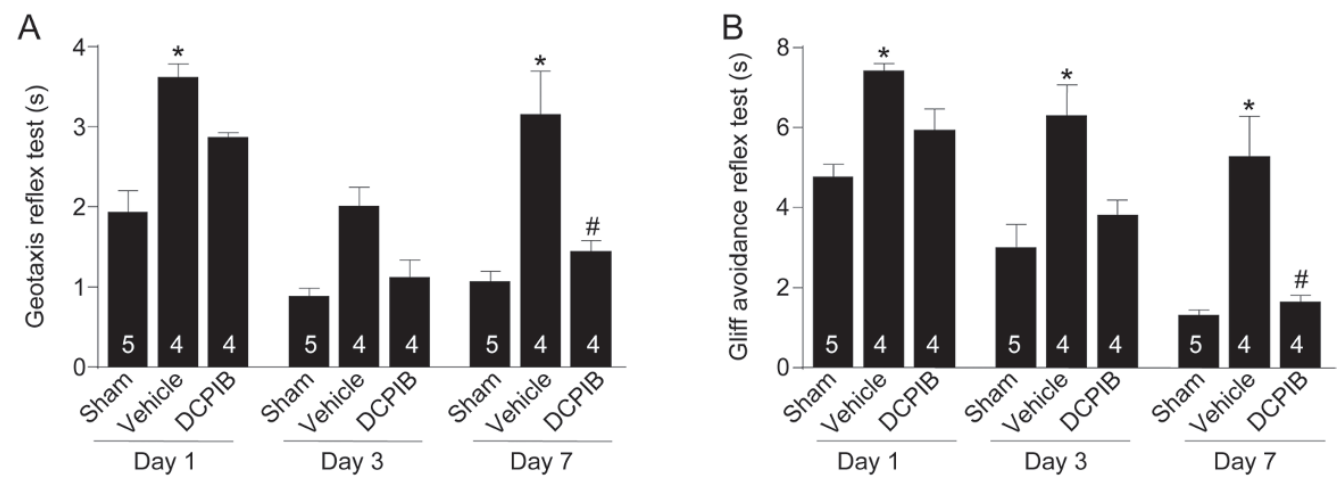

Figure 5. Neurobehavioural performance after $\mathrm{HI}$ is improved with DCPIB treatment. (A) Summary of geotaxis reflex test on Day 1, Day 3, and Day 7 (when mouse pups were P8, P10, and P14, respectively) across animals from all groups: sham ( $n=5)$, control HI ( $n=4)$, and DCPIB-treated HI ( $n=4)$. Animals in the control $\mathrm{HI}$ group consistently had a slower reflex, either significant $\left({ }^{*} P<0.05\right)$ or clearly trending towards significance. Note that comparisons were made between groups on each respective day (compared to the sham group). The general trend is that DCPIB-treatment improves geotaxis reflex towards that of sham animals. (B) Summary of cliff avoidance reflex test on Day 1, Day 3, and Day 7 (when mouse pups were P8, P10, and P14, respectively) across animals from all groups: sham $(n=5)$, control $\mathrm{HI}(n=4)$, and DCPIB-treated $\mathrm{HI}(n=4)$. Animals in the control $\mathrm{HI}$ group consistently had a significant slower reflex $\left({ }^{*} P<0.05\right)$. Note that comparisons were made between groups on each respective day (compared to the sham group). The general trend is that DCPIB-treatment improves cliff avoidance reflex towards that of sham animals. (One-way ANOVA with Bonferonni multiple comparison test).

note that at this time point, DCPIB-treated HI group animals still performed worse than sham group animals (at $1.07 \pm 0.13 \mathrm{~s}$; $P<0.05)$; nevertheless, the difference was minimal and the trend of improvement was clearly present. Similarly, animals in the DCPIB-treated group also demonstrated improvement in cliff avoidance reflex at Day 7; reflex time was reduced from $5.27 \pm 1.02 \mathrm{~s}$ (control HI group) to $1.64 \pm 0.17 \mathrm{~s}$ (DCPIB-treated HI group; $P<0.05)$. However, although evidently trending towards the same reflex levels as animals in the sham group (at $1.31 \pm 0.14 \mathrm{~s}$ ), pups in the DCPIB-treated HI group remained significantly slower $(P<0.05)$. Nevertheless, we provide the first evidence that a single injection of DCPIB $\sim 20$ min prior to $\mathrm{CCAO}$ can elicit a lasting neuroprotective effect that ultimately improves neurobehavioural performance.

In our previous study, we focused on the in vitro effects of DCPIB by demonstrating the effects of DCPIB on OGD and intracellular chloride concentration ${ }^{[17]}$. Specifically, we showed that the resting $\left[\mathrm{Cl}^{-}\right]_{\mathrm{i}}$ significantly increased following OGD in the PC12 cell line, and that DCPIB $(10 \mu \mathrm{mol} / \mathrm{L})$ reduced this influx. Furthermore, we demonstrated that treating PC12 cells with DCPIB significantly reduced cell death induced by OGD, and ultimately increased cell survival. In addition, DCPIB alone did not show any toxicity on cultured cells, which was suggestive of safety ${ }^{[17]}$. This in vitro work gave evidence that inhibition of the swelling-induced $\mathrm{Cl}^{-}$current $I_{\mathrm{Cl}, \text { swell }}$ can ameliorate OGD-induced injury. Recently, we showed that DCPIB inhibited the swelling-induced $\mathrm{Cl}^{-}$current $I_{\mathrm{Cl}, \text { swell }}$, and consequently suppressed the malignant cellular functions (ie, proliferation, migration, and invasion) of glioblastoma cell lines (ie, U87, U251) ${ }^{[18]}$. This suggests that DCPIB is an effective swelling-induced $\mathrm{Cl}^{-}$current $I_{\mathrm{Cl} \text {,swell }}$ antagonist across multiple cell types. In our present study, we examined the in vivo effects of DCPIB using a neonatal mouse HI model. Specifically, we assessed the behavioural, histological, and morphological aspects of the HI insult with or without DCPIB treatment. This is unknown in the literature and represents a knowledge gap, because inhibition of the swelling-induced $\mathrm{Cl}^{-}$current $I_{\mathrm{Cl} \text {,swell }}$ can potentially be exploited as an effective therapeutic strategy for neonatal HI brain injury. Moreover, DCPIB has been reported to be effective in reducing the infarct volumes of adult MCAO model ${ }^{[16]}$, which further articulates the broad implications and therapeutic feasibility of DCPIB in ischemia. However, in adult brain ischemia, DCPIB only demonstrated neuroprotection when given intracisternally, but not intravenously. This was in part due to an impedance of drug delivery by the developed blood brain barrier $(\mathrm{BBB})^{[16]}$. In our previous ${ }^{[17]}$ and current studies, however, we showed evidence that intraperitoneal administration of DCPIB was able to cross the immature BBB of the perinatal or neonatal brain. That is, DCPIB treatment exerted neuroprotection during $\mathrm{HI}$ insults by significantly reducing brain damage and improving neurobehavioural outcomes. The BBB in neonates may be immature and undergoing developmental changes, thus favouring drug penetration into the neonatal brain, especially during ischemic conditions which can disrupt the BBB integrity ${ }^{[27]}$. Here, we showed critical in vivo evidence for the pathophysiological role of the swelling-induced $\mathrm{Cl}^{-}$current $I_{\mathrm{Cl} \text {,swell }}$ in the $\mathrm{HI}$ brain injury. Moreover, we provided important findings for potential drug development of swelling-induced $\mathrm{Cl}^{-}$current $I_{\mathrm{Cl}, \text { swell }}$ antagonists such as DCPIB as a readily administrable drug for prevention and treatment of the $\mathrm{HI}$ brain injury. In conclusion, we established that the effects mediated by the swelling-induced $\mathrm{Cl}^{-}$current $I_{\mathrm{Cl}, \text { swell }}$ represent a non-glutamate mechanism of the neonatal $\mathrm{HI}$ brain injury (Figure 6). Thus, the swelling-induced $\mathrm{Cl}^{-}$current $I_{\mathrm{Cl}, \text { swell }}$ is exploitable as a drug target (by using selective potent antagonists; eg, DCPIB) in order to prevent and treat human neonatal $\mathrm{HI}$ brain injury in addition to its consequential neurological disorders such as 


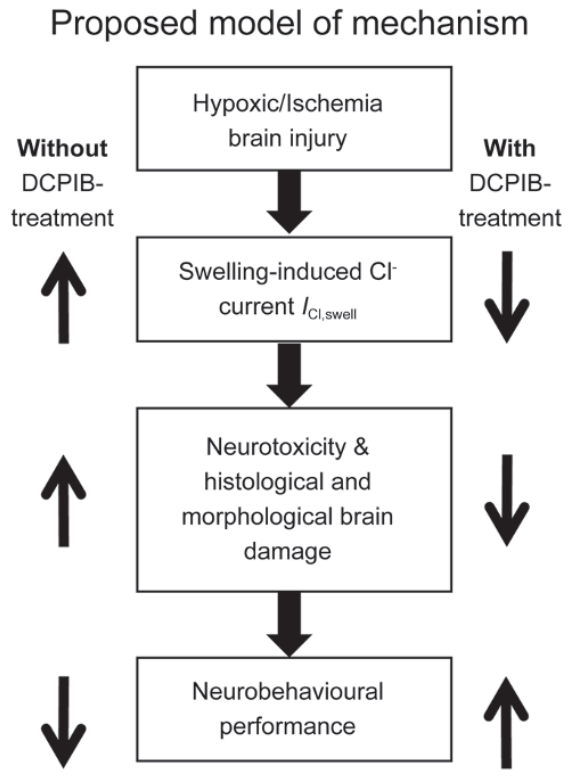

Figure 6. Schematic of proposed model of mechanism underlying the swelling-induced $\mathrm{Cl}^{-}$current $I_{\mathrm{Cl}, \text { swell }}$ in $\mathrm{HI}$ brain injury. Following hypoxic and ischemic insult, there is aberrant activity of the swelling-induced $\mathrm{Cl}^{-}$ current $I_{\mathrm{Cl}, \text { swell }}$. This can lead to neuronal death and result in brain damage. Ultimately, neurobehavioural performance may be negatively affected. However, this outcome can be reversed with treatment using the specific swelling-induced $\mathrm{Cl}^{-}$current $I_{\mathrm{Cl}, \text { swell }}$ antagonist DCPIB. Aberrant swellinginduced $\mathrm{Cl}^{-}$current $I_{\mathrm{Cl}, \text { swell }}$ activity is reduced. Consequently, there is less neuronal death and brain damage, which would contribute to improved neurobehavioural performance.

hypoxic-ischemic encephalopathy and cerebral palsy.

\section{Acknowledgements}

This work was supported by the following grants: Natural Sciences and Engineering Research Council of Canada (NSERC) Discovery Grants to Hong-shuo SUN (RGPIN-2016-04574); a Canadian Institutes of Health Research (CIHR) China-Canada Joint Health Research Initiative (CIHR, FRN \#132571) to Hong-shuo SUN; Natural Sciences and Engineering Research Council of Canada (NSERC) Discovery Grants to Zhong-ping FENG (RGPIN-2014-06471) and to Hong-shuo SUN (RGPIN2016-04574); the NSFC-CIHR Joint Health Research Initiative Proposal (No 81361128011) to Guan-lei WANG; a NSERC Postgraduate Scholarship-Doctoral to Raymond WANG.

\section{Author contribution}

Hong-shuo SUN and Zhong-ping FENG developed the concepts and designed the study. Raymond WONG, Ahmed ABUSSAUD, Joseph WH LEUNG, Bao-feng XU, Sammen HUANG, and Fei-ya LI performed experiments and analyzed the data; Raymond WONG and Hong-shuo SUN wrote the manuscript; all authors discussed the results and commented on the manuscript.

\section{References}

1 Vannucci SJ, Hagberg $\mathrm{H}$. Hypoxia-ischemia in the immature brain. J
Exp Biol 2004; 207: 3149-54.

2 Vannucci SJ. Cerebral ischemia and the developing brain: introduction. Stroke 2007; 38: 723.

3 Vannucci RC. Hypoxic-ischemic encephalopathy. Am J Perinatol 2000; 17: 113-20.

4 Nelson KB, Lynch JK. Stroke in newborn infants. Lancet Neurol 2004; 3: 150-8.

5 Nelson KB. Perinatal ischemic stroke. Stroke 2007; 38: 742-5.

6 Little WJ. On the influence of abnormal parturition, difficult labours, premature birth, and asphyxia neonatorum, on the mental and physical condition of the child, especially in relation to deformities. Clin Orthop Relat Res 1966; 46: 7-22.

7 Kruse M, Michelsen SI, Flachs EM, Bronnum-Hansen H, Madsen M, Uldall P. Lifetime costs of cerebral palsy. Dev Med Child Neurol 2009; 51: 622-8.

8 Centers for Disease Control and Prevention (CDC). Economic costs associated with mental retardation, cerebral palsy, hearing loss, and vision impairment. MMWR Morb Mortal Wkly Rep 2004; 53: 57-9.

9 de Vries LS, Jongmans MJ. Long-term outcome after neonatal hypoxic-ischaemic encephalopathy. Arch Dis Child Fetal Neonatal Ed 2010; 95: F220-F224.

10 Besancon E, Guo S, Lok J, Tymianski M, Lo EH. Beyond NMDA and AMPA glutamate receptors: emerging mechanisms for ionic imbalance and cell death in stroke. Trends Pharmacol Sci 2008; 29: 268-75.

11 Guan YY, Wang GL, Zhou JG. The CIC-3 $\mathrm{Cl}^{-}$channel in cell volume regulation, proliferation and apoptosis in vascular smooth muscle cells. Trends Pharmacol Sci 2006; 27: 290-6.

12 Kim SY, Shin DH, Kim SJ, Koo BS, Bae CD, Park J, et al. Chloride channel conductance is required for NGF-induced neurite outgrowth in PC12 cells. Neurochem Int 2010; 56: 663-9.

13 Duan D, Winter C, Cowley S, Hume JR, Horowitz B. Molecular identification of a volume-regulated chloride channel. Nature 1997; 390: 417-21.

14 Decher N, Lang HJ, Nilius B, Bruggemann A, Busch AE, Steinmeyer $\mathrm{K}$. DCPIB is a novel selective blocker of $I_{\mathrm{Cl}, \text { swell }}$ and prevents swelling induced shortening of guinea-pig atrial action potential duration. $\mathrm{Br} \mathrm{J}$ Pharmacol 2001; 134: 1467-79.

15 Inoue $\mathrm{H}$, Okada $\mathrm{Y}$. Roles of volume-sensitive chloride channel in excitotoxic neuronal injury. J Neurosci 2007; 27: 1445-55.

16 Zhang Y, Zhang H, Feustel PJ, Kimelberg HK. DCPIB, a specific inhibitor of volume regulated anion channels (VRACs), reduces infarct size in MCAO and the release of glutamate in the ischemic cortical penumbra. Exp Neurol 2008; 210: 514-20.

17 Alibrahim A, Zhao LY, Bae CY, Barszczyk A, Sun CLF, Wang GL, et al. Neuroprotective effects of volume-regulated anion channel blocker DCPIB on neonatal hypoxic-ischemic injury. Acta Pharmacol Sin 2013; 34: 113-8.

18 Wong R, Chen W, Zhong X, Rutka JT, Feng ZP, Sun HS. Swellinginduced chloride current in glioblastoma proliferation, migration and invasion. J Cell Physiol 2017; 233: 363-70.

19 Sun HS, Jackson MF, Martin LJ, Jansen K, Teves L, Cui H, et al. Suppression of hippocampal TRPM7 protein prevents delayed neuronal death in brain ischemia. Nat Neurosci 2009; 12: 1300-7.

20 Lubics A, Reglodi D, Tamas A, Kiss P, Szalai M, Szalontay L, et al. Neurological reflexes and early motor behavior in rats subjected to neonatal hypoxic-ischemic injury. Behav Brain Res 2005; 157: 15765.

21 Chen W, Xu B, Xiao A, Liu L, Fang X, Liu R, et al. TRPM7 inhibitor carvacrol protects brain from neonatal hypoxic-ischemic injury. Mol Brain 2015; 8: doi:10.1186/s13041-015-0102-5.

22 Xiao AJ, Chen W, Xu B, Liu R, Turlova E, Barszczyk A, et al. Marine 
compound xyloketal B reduces neonatal hypoxic-ischemic brain injury. Mar Drugs 2014; 13: 29-47.

23 Xu B, Xiao AJ, Chen W, Turlova E, Liu R, Barszczyk A, et al. Neuroprotective effects of a PSD-95 inhibitor in neonatal hypoxicischemic brain injury. Mol Neurobiol 2016; 53: 5962-70.

24 Huang S, Turlova E, Li F, Bao MH, Szeto V, Wong R, et al. Transient receptor potential melastatin 2 channels (TRPM2) mediate neonatal hypoxic-ischemic brain injury in mice. Exp Neurol 2017; 296: 32-40.

25 Huang S, Wang H, Turlova E, Abussaud A, Ji X, Britto LR, et al. GSK$3 \beta$ inhibitor TDZD-8 reduces neonatal hypoxic-ischemic brain injury in mice. CNS Neurosci Ther 2017; 23: 405-15.

26 Wang $\mathrm{H}$, Huang S, Yan K, Fang X, Abussaud A, Martinez A, et al. Tideglusib, a chemical inhibitor of GSK3 $\beta$, attenuates hypoxicischemic brain injury in neonatal mice. Biochim Biophys Acta 2016; 1860: 2076-85.

27 Sun HS, Xu B, Chen W, Xiao A, Turlova E, Alibraham A, et al.
Neuronal $\mathrm{K}_{\text {ATP }}$ channels mediate hypoxic preconditioning and reduce subsequent neonatal hypoxic-ischemic brain injury. Exp Neurol 2015; 263: 161-71.

28 Turlova E, Bae CY, Deurloo M, Chen W, Barszczyk A, Horgen FD, et al. TRPM7 regulates axonal outgrowth and maturation of primary hippocampal neurons. Mol Neurobiol 2014; 53: 595-610.

29 Wong R, Chen W, Zhong X, Rutka JT, Feng ZP, Sun HS. Swellinginduced chloride current in glioblastoma proliferation, migration and invasion. J Cell Physiol 2017; 233: 363-70.

30 Chen WL, Turlova E, Sun CL, Kim JS, Huang S, Zhong X, et al. Xyloketal B suppresses glioblastoma cell proliferation and migration in vitro through inhibiting TRPM7-regulated PI3K/Akt and MEK/ERK signaling pathways. Mar Drugs 2015; 13: 2505-25.

31 Chen WL, Barszczyk A, Turlova E, Deurloo M, Liu B, Yang BB, et al. Inhibition of TRPM7 by carvacrol suppresses glioblastoma cell proliferation, migration and invasion. Oncotarget 2015; 6: 16321-40. 